\title{
2020 ISH GLOBAL HYPERTENSION GUIDELINES ISH returns to the fray with its own Global Guidelines
}

\author{
JOHN CHALMERS \\ The George Institute for Global Health; \\ University of New South Wales \\ Sydney, Australia
}

DOI: $10.30824 / 2006-11$

A welcome and successful return by the ISH to the crowded field of Guidelines for the Management of Hypertension!

The Mission of the ISH is reflected in its own Guidelines!

Congratulations to the current Council of the ISH for biting the bullet and re-entering the field of guidelines for the management of hypertension ${ }^{1}$ 2 and doing so in a unique fashion suited to the particular mission of the ISH in the crowded space of guidelines commissioned by societies of hypertension or allied disorders. Because the ISH had traditionally accepted responsibility for advising on the management hypertension at the global level, including the vast mass of patients living in disadvantaged communities spread around the world, with large concentrations in Africa, Asia and Latin America. To that end, the ISH had partnered with the World Health Organisation to produce a series of Guidelines known as the "WHO-ISH Guidelines" or "Statements" from the throughout the !970s, through the 1980s and 1990s to 2003, before vacating the field (except for one joint effort with the American Society of Hypertension in 2014, ${ }^{3}$ ), as the task became increasingly laborious, and was taken over by continental and national societies with greater resources and ease of communication at the geographic level. However the SPRINT trial ${ }^{4}$ published in 2015, stimulated a spate of new guidelines from North America, Europe and Asia ${ }^{5}$ $6,7,8,9$ and it is gratifying to see this new set of ISH Guidelines contributing, and bringing its own unique perspective. These ISH guidelines are unusual in separating their recommendations into those that are "ESSENTIAL" and thus applicable to all patients, including those in disadvantaged communities, or in Lower and Middle Income Countries (LMIC), and those that are "OPTIMAL" and thus more suited for use in high income countries (HIC) that can afford more expensive procedures and equipment. This simplifies the tasks for community doctors, nurses, and health care workers, practicing in less affluent societies, who for example would not need to measure blood pressure (BP) using Automated Unattended Blood Pressure Measurement (AOBPM). And even more, it simplifies the regimen for treatment as clearly shown in differentiating the choices for drug treatment (see Figure 1), between those in high income situations, who go down the OPTIMAL path and are offered a progression of single pill combinations (SPC), whereas those in LMIC go down the ESSENTIAL path and are basically offered whatever drugs are available locally, to try and build rational combinations when SPC are not available.

\section{Some Notable Features}

First, congratulations on keeping the guidelines down to just over $\mathbf{2 0}$ pages! including the references, and with good tables and figures. That makes such a difference for the poor, overworked reader, when compared to say the most recent European Guidelines at 90 pages or the American Guidelines at 170 pages. Different missions and different target audiences, but for a physician or nurse in rural practice in India, or Vietnam, or Nigeria or Brazil, that would make a very welcome difference!! 
Second, a bouquet for backing combination treatment without any hesitation in the OPTIMAL pathway, and with sensible compromise in the ESSENTIAL pathway, and for recommending SPC therapy for all patients who warrant drug therapy, including for the initiation of therapy. Thus, for patients in high income situations, drug therapy starts and continues with SPC, initially low-dose combinations of two drugs, usually at half the standard dose, followed if necessary by dual full dose SPC, and then if necessary by a triple combination, whereas patients in less fortunate situations make do with whatever drugs are available to get as close to the ideal as possible, with good diagrams to illustrate both pathways (see Figure 1).

\section{Essential}

- Use whatever drugs are available with as many of the ideal characteristics (see Table 9) as possible.

- Use free combinations if SPCS are not available or unaffordable

- Use thiazide diuretics if thiazide-like diuretics are not available

- Use alternative to DHPCCBs if these are not available or not tolerated (i.e. Non-DHPCCBs: diltiazem or verapamil).
Ideally Single Pill

Combination

Therapy (SPC

\section{Optimal}
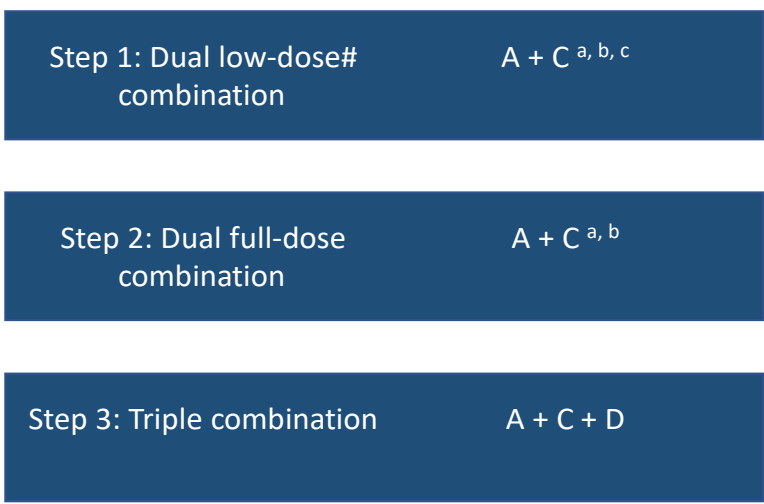

Step 4: (Resistant

Hypertension)

Triple Combination

+ Spironolactone or other drug*

\section{Essential \\ and \\ Optimal}

Consider beta-blockers at any treatment step when there is a specific indication for their use, e.g. heart failure, angina, post-MI, atrial fibrillation, or younger women with, or planning pregnancy. a) Consider monotherapy in low risk grade 1 hypertension or in very old ( $\geq 80 \mathrm{yrs}$ ) or frailer patients.

b) Consider $A+D$ in post-stroke, very elderly, incipient heart failure or CCB intolerance.

c) Consider A + C or C + D in black patients.

d) Caution with spironolactone or other potassium sparing diuretics when estimated GFR $<45 \mathrm{ml} / \mathrm{min} / 1.73 \mathrm{~m} 2$ or $\mathrm{K}+>4.5 \mathrm{mmol} / \mathrm{L}$.

$A=A C E-I n h i b i t o r$ or ARB (Angiotensin Receptor Blocker)

$\mathrm{C}=\mathrm{DHP}-\mathrm{CCB}$ (Dihydropyridine -Calcium Channel Blocker)

$D=$ Thiazide-like diuretic

Supportive references: $A+C, 69,70$ Spironolactone, ${ }^{71}$ Alpha-blocker, ${ }^{72} C+D^{73}$.

* Alternatives include: Amiloride, doxazosin, eplerenone, clonidine or beta-blocker. \# low-dose generally refers to half of the maximum recommended dose

RCT-based benefits between ACE-I's and ARB's were not always identical in different patient populations. Choice between the two classes of RAS-Blockers will depend on patient characteristics, availability, costs and tolerability

\section{Figure 1: ISH Core Drug Treatment Strategy.}

Adapted from Hypertension. 2020;75:1334-1357, Journal of Hypertension. 2020, 38(6):982-1004 
The Guidelines are also unusual in being written by the members of the Council, that is by the members of the governing body of the organisation. While members of a Governing Council or Board are normally responsible for setting the organisation's mission and strategy, it must be unusual for the members of the supreme body in any major organisation, to be those asked to actually write the detailed proposal for how to improve the mission and the daily business of the organisation, and even more unusual to see something sensible emerge! But, I have to say it worked well in this instance, probably because the ISH tries hard to make sure its Council does consist of individuals, each of whom does understand hypertension, and who between them do represent the many disciplines involved and the many large human populations with hypertension on our planet, including that disadvantaged majority that lives in LMIC!

The Measurement of $\mathrm{BP}$ recommended is pretty standard, but very thoroughly described, including out of office BP, with AOBPM only recommended for OPTIMAL situations. Hypertension is defined as $\geq 140 / 90 \mathrm{mmHg}$ when measured in the office or clinic, and the target BP is set at $\leq 140 / 90 \mathrm{mmHg}$ for low risk patients with treatment starting with lifestyle modifications, and at $\leq 130 / 80 \mathrm{mmHg}$ for patients with significant risk factors such as overweight-obesity or diabetes, or Hypertension

The classification of Hypertension, based on office BP (Based on figure1)

\section{$\mathbf{m m H g}$}

Normal BP

$<130 / 85$

High Normal

$130-139 / 85-89$

Definition of Hypertension $\geq 140 / 90$

$140-159 / 90-99$

Grade 2 Hypertension

the $w \geq 160 / 100$

Mediated Organ Damage (HMOD), with easy to read tables and figures to assist the reader. But it is interesting that the authors recommend a target of $\leq 140 / 90 \mathrm{mmHg}$ for those with significant comorbidities over 65 years, instead of $\leq 130 / 80 \mathrm{mmHg}$, which suggests that in effect like many other authorities around the world, they have only accepted part of the messages from SPRINT, and were not convinced by the finding that those over the age of 75 did best with the more intensive BP regime, but there is of course much other evidence to bear on this much debated topic. There are simple but comprehensive sections on lifestyle measures, and importantly on adherence to recommended drug therapy, with a good reminder of the stark findings from the PURE study that the rule of halves still holds and that more patients fail to take their tablets than those that do actually swallow them!

But I am full of admiration that quite apart from these main messages and sections, the authors have succeeded in fitting in useful sections on a large number of important conditions, all within their $20+$ pages!

These include sections on "Common and other comorbidities and complications" which go beyond the expected conditions of Coronary disease, Stroke, Heart Failure, Chronic Kidney Disease, and Diabetes, to include Inflammatory Diseases, Psychiatric Diseases, and quite considerable sections on Secondary Hypertension, on Exacerbators and Inducers of Hypertension, and on Resistant Hypertension. But there are also quite useful pages devoted to Hypertension in Pregnancy, to Hypertensive Emergencies and to Ethnicity, Race and Hypertension. And there are schematic single page diagrammatic summaries of the whole of the ESSENTIAL pathway and the OPTIMAL pathway each of which could easily be printed out onto two sides of an A5 card for carrying on busy daily rounds in hospital or community practice.

\section{A Bouquet to the authors for a very valuable contribution!}


1. Unger T, Borghi C, CharChar F, et al. 2020 International Society of Hypertension global hypertension practice guidelines. Journal of Hypertension 2020;38:982-1004. doi: 10.1097/HJH.0000000000002453

2. Unger T, Borghi C, Charchar F, et al. 2020 International Society of Hypertension Global Hypertension Practice Guidelines. Hypertension 2020;75:00-00.doi:10.1161/ HYPERTENSIONAHA.120.15026

3. Weber MA, Schiffrin EL, White WB, et al. Clinical Practice Guidelines for the Management of Hypertension in the Community. A Statement by the American Society of Hypertension and the International Society of Hypertension. J Hypertension 2014;32:315 doi: $10.1097 / \mathrm{HJH} .0000000000000065$

4. Wright JT, Williamson JD, Whelton PK et al for the SPRINT Research Group. A randomised trial of intensive versus standard blood pressure control. N Engl J Med 2015;373:21032116. doi: 10.1056/NEJMoa1511939

5. Whelton PK, Carey RM, Aronow WS et al (2017) ACC/AHA/AAPA/ABC/ACPM/AGS/APhA/ ASH/ASPC/NMA/PCNA Guidelines for the prevention, detection, evaluation and management of high blood pressure in adults: a report of the American College of Cardiology/American Heart Association Task Force on Clinical Practice Guidelines. J Am Coll Cardiol. 2018;71:e127e248 doi: 10.1016/j.jacc.2017.11.006 [published Online First 2017/11/18]

6. Williams B, Mancia G, Spiering W et al. 2018 ESC/ESH Guidelines for the management of arterial hypertension. Journal of Hypertension 2018; 36:1953-2041. doi: 10.1097/HJH.0000000000001940

7. Nerenberg K, Zarnke KB, Leung AA et al, Hypertension Canada's 2018 Guidelines for Diagnosis, Risk Assessment, Prevention, and Treatment of Hypertension in Adults and Children. Canadian Journal of Cardiology 2018; 34:506-525. doi: 10.1016/j.cjca.2018.02.022

8. The National Institute for Health and Care Excellence. Hypertension in Adults: diagnosis and management. NICE guideline 136.London. National Institute for Health and Care Excellence 2019. https//www.nice.org.uk.guidance/ng136

9. The Japanese Society of Hypertension Guidelines for the Management of Hypertension (JSH 2019). Hypertension Research 2019;42:1235148 doi:10.1038/s41440-019-0284-9

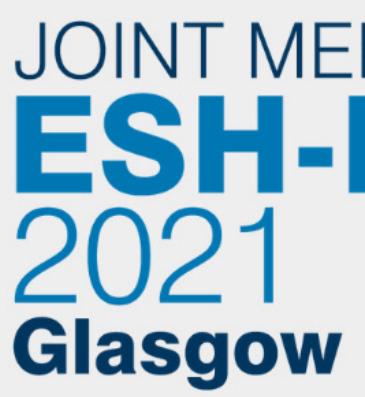

April 11-14, 2021 Scottish Event Campus Glasgow, United Kingdom www.hypertension2021.org
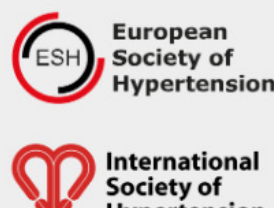

Hypertension

$\downarrow \mathrm{BHS}$ hub

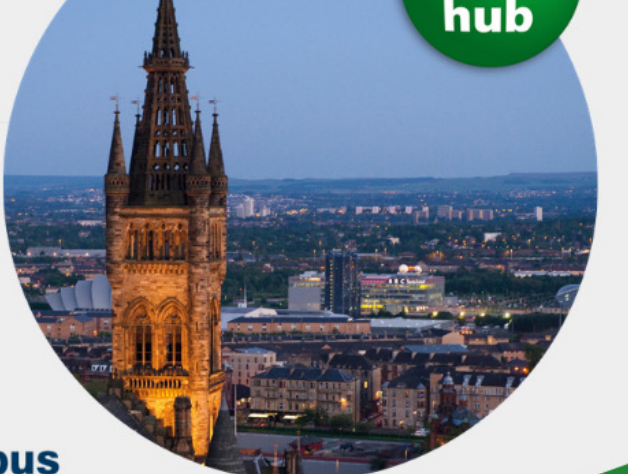
B.HS

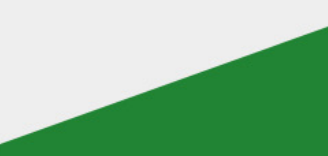

